\title{
ROTATION IN PROMINENCES
}

\author{
M. LIGGETT and H. ZIRIN \\ Big Bear Solar Observatory, California Institute of Technology, Pasadena, CA 91125, U.S.A.
}

(Received 12 October, 1983; in revised form 25 January, 1984)

Abstract. We have studied rotation in non-eruptive limb prominences; in most cases dopplergrams could be used to confirm proper motion measurements. In some cases part of the prominence rotates; in the others, the entire body is in rotation. Velocities of $15-75 \mathrm{~km} \mathrm{~s}^{-1}$ are found. Of fifty-one prominences studied in 1978 , five showed rotation.

\section{Introduction}

At the Nobel Symposium in Capri in 1968, Öhman (1968) discussed several cases where spectrographic data indicated rotational motion in quiescent prominences. He later (1969) presented additional spectral data and developed models for the various spectral patterns. Rompolt (1975), in a slightly different vein, developed anticipated spectral shapes from various possible rotational prominence motions.

In November of 1977 at Big Bear, we noted an interesting circular motion in a large, relatively quiescent prominence on the east limb of the Sun. The area of motion involved less than one quarter of the prominence and appeared to be a large scale version of Ohman's 'smoke-ring' (1969). A film of this prominence was shown at IAU Colloq. 44 in Oslo. Unfortunately no Doppler scans of that event were obtained, so that true rotation (as contrasted to phase brightening) could be demonstrated. Subsequent observations provided several examples of radial velocity (from line-shifted frames) and proper motion, confirming that physical rotation was taking place.

Rotation in prominences is somewhat difficult to understand in view of our concept of prominences suspended in magnetic fields. If the field threads the material, then rotation must wind up the magnetic field, transferring energy to the field and slowing down the rotation. In the cases we have studied, rotation continues through several turns, which raises real problems. The rotation discussed here is different from that often seen in erupting prominences. In quiescents the process is continuous and the eddy size remains constant or decreases; in eruptives, the eddy size grows rapidly. Malville and Toot (1982) have reported transient motion and impulsive brightening in prominences; these are discontinuous and probably a different phenomenon.

\section{Observations}

We studied the prominences observed during 1978 (a year in which we concentrated on limb prominences) and picked out prominences showing apparent motion. Typically the prominences observed showed horizontal motion, and a few showed upward or downward flow. Five of the fifty-one prominences examined revealed localized or 
general rotational motion. We have studied in detail the three of these with the most adequate data, as well as the above-mentioned case of November, 1977. In each case time-lapse films were obtained for at least $8 \mathrm{hr}$. Despite the considerable motion, none erupted during that day; all showed some trace of prominence material at the limb the next day. $\mathrm{H} \alpha$ frames with $0.25 \AA$ bandpass (except 2 August, 1978 , when $0.5 \AA$ was used) were obtained every 15 to $18 \mathrm{~s}$. For all but one, Doppler scans $(\Delta \lambda=0.25 \AA)$ were run approximately every hour.

Motion in the plane of the sky was measured by projecting $35 \mathrm{~mm}$ negatives and sketching specific knots. The number of measurements and the timing between them varied, depending on seeing from frame to frame and the 'lifetime' of the knot. (A knot typically was followed about $300 \mathrm{~s}$.) Sometimes the knots appeared to accelerate or decelerate.

For the three samples with Doppler scans, cancelled velocity photos were produced by Leighton's (1959) method giving contrasting black and white regions where there is receding and advancing material respectively. (See the $b$ columns of Figures 3 and 5.) These dopplergrams were made for $0.25,0.5,0.75$, and $1.0 \AA$ off the center-line of $\mathrm{H} \alpha$ (6563 $\AA$ ). Photometric measurements of knots in the wavelength scans provided coarse line profiles giving the line shifts and hence radial velocity, which was of the same magnitude as the transverse motion.

Table I gives the pertinent data for the prominences studied. To give one an idea of the portion of the prominence being affected by this rotation, the height of the prominence and the height and width of the rotating region are given. Doppler rotation rates are from the photometric measurements.

TABLE I

Prominence data

\begin{tabular}{|c|c|c|c|c|c|c|c|}
\hline \multirow[t]{2}{*}{ Date } & \multirow[t]{2}{*}{$\begin{array}{l}\text { Prom. } \\
\text { height } \\
(\times 10000 \mathrm{~km})\end{array}$} & \multirow{2}{*}{$\begin{array}{l}\text { Rotating } \\
\text { region } \\
\text { size } \\
(\times 1000 \mathrm{~km})\end{array}$} & \multicolumn{3}{|c|}{$\begin{array}{l}\text { Rotation rate - } \\
\text { plane of sky } \\
\left(\mathrm{km} \mathrm{s}^{-1}\right)\end{array}$} & \multicolumn{2}{|c|}{$\begin{array}{l}\text { Rotation rate } \\
\text { line of sight } \\
\left(\mathrm{km} \mathrm{s}^{-1}\right)\end{array}$} \\
\hline & & & Max & Min & Ave & Red & Blue \\
\hline 26 Nov. 77 & 4.4 & $3 \times 7$ & 75 & 30 & 55 & no data & \\
\hline 31 Jul. 78 & 3 & $1.5 \times 9$ & 23 & 15 & 18 & 4 & 8 \\
\hline 2 Aug. 78 & 6 & $10 \times 20$ & 30 & 15 & 20 & 10 & 9 \\
\hline $\begin{array}{l}18 \text { Aug. } 78^{a} \\
\text { (16:00 UT) }\end{array}$ & 13 & $18 \times 73$ & 55 & 32 & 44 & 40 & 28 \\
\hline $\begin{array}{l}18 \text { Aug. } 78^{a} \\
\text { (18:00 UT) }\end{array}$ & 13 & $27 \times 59$ & 43 & 12 & 21 & 23 & 26 \\
\hline $\begin{array}{l}18 \text { Aug. } 78^{a} \\
(20: 00 \text { UT })\end{array}$ & 14 & $30 \times 104$ & 72 & 21 & 41 & 21 & 23 \\
\hline
\end{tabular}

a This event shows different morphologies; thus more than one set of data is included. 
Following is a brief description of each event:

\section{November, 1977 (Figures 1 and 2)}

This prominence was the first in which we noted rotation. It was not obvious to the eye, but films showed that one section of an otherwise quite stable prominence on the northeast limb was rotating rapidly. Unfortunately the observer (HZ) forgot to take the usual Doppler scans, so we cannot confirm actual mass motions, but the rotation is very well marked. Differencing of center-line frames separated by various time intervals (Figure 1; b, c, and d) shows how the prominence changes rapidly in the rotating area while the rest remains gray (unchanged). Figure 2 shows an $\mathrm{H} \alpha$ frame later in the day, when a complete eddy could be seen.

As can be seen from the arrows and cancellations in Figure 1 (bright means more emission in the second frame, black means less) there was upflow at the left of the arch, flowing over the top and returning below. The return is better seen in Figure 2. Rotation occurred in outbreaks lasting about an hour; each outbreak started from the same general area and broke down into four or five smaller eddies. The scale of the main eddy was around $9000 \mathrm{~km}$. Occasionally bright knots moved along the inner edge of the main eddy. Although we cannot follow a single knot through an entire roll, we can estimate the rollover time from the size of the main eddy and the velocity of the knot for the $100^{\circ}$ or so over which we can follow it. This time was about half an hour, giving a proper motion of $25 \mathrm{~km} \mathrm{~s}^{-1}$ which agrees with individual knot measurements. The rollover time is long enough for the turbulent viscosity to subdue each outbreak. There were about three rollovers in each outbreak.

The prominence did not erupt. It came onto the disc as a large, broad, diffuse filament. On 2 December it began to consolidate into a compact, dense structure which made a complete transit of the disc. We see no evidence that the rotating part was a separate prominence projected against it.

\section{July, 1978 (Figure 3.1)}

This was a stable hedgerow prominence on the southeast limb, with a small recurrent rotation (marked by an arrow) near the central trunk. Periods of rotation occurred at $\sim 16: 15,18: 05$, and $20: 25$, in each case with proper motion averaging $18 \mathrm{~km} \mathrm{~s}^{-1}$. The last outbreak was the strongest and continued to the end of the day $(01: 50 \mathrm{UT}$, 1 August, 1978). The Doppler cancellations (Figure 3.1b) indicate motions of about $6 \mathrm{~km} \mathrm{~s}^{-1}(1 / 4 \AA)$. Combining these with the proper motion $\left(18 \mathrm{~km} \mathrm{~s}^{-1}\right)$ we find a total rotational velocity of about $19 \mathrm{~km} \mathrm{~s}^{-1}$ about an axis $45^{\circ}$ to the left of the line of sight in a plane tangent to the limb.

On the next day high resolution coverage showed a high wispy 'cloud' from which material poured down towards a small triangular base. Patrol films showed that the filament existed for a full disc transit. 


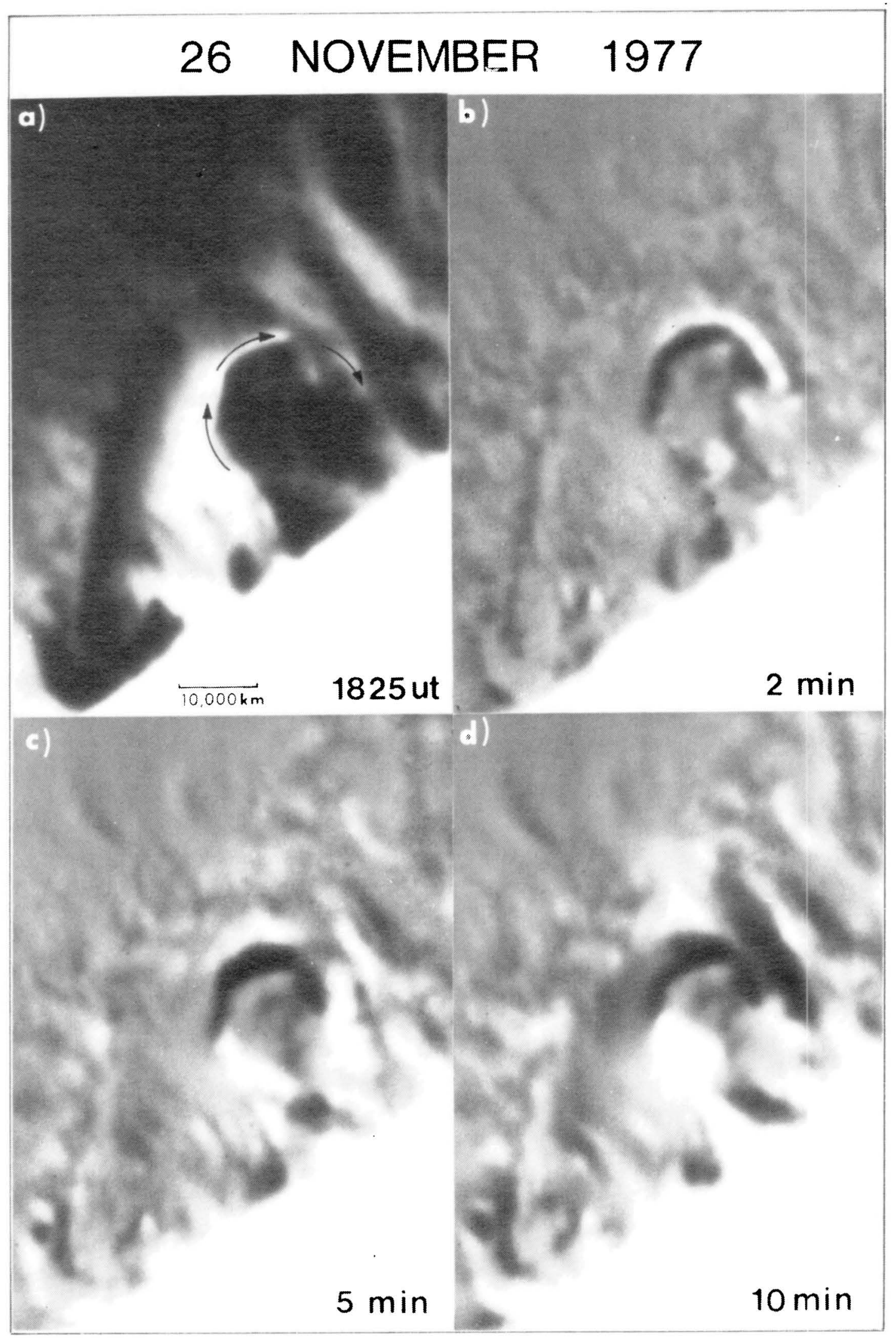

Fig. 1. Prominence of 26 November, 1977: (a) H $\alpha$ line-center 18:25 UT, and (b), (c), and (d) time subtractions of center-line frames at intervals of 2,5 , and $10 \mathrm{~min}$ with $t_{0}=18: 25$ UT. Black indicates the material brighter at the beginning and white, brighter later. Grey indicates no change. These are not Doppler subtractions! Arrows indicate the direction of apparent proper motion. Material entered at the left of the arch and came down at the right. 


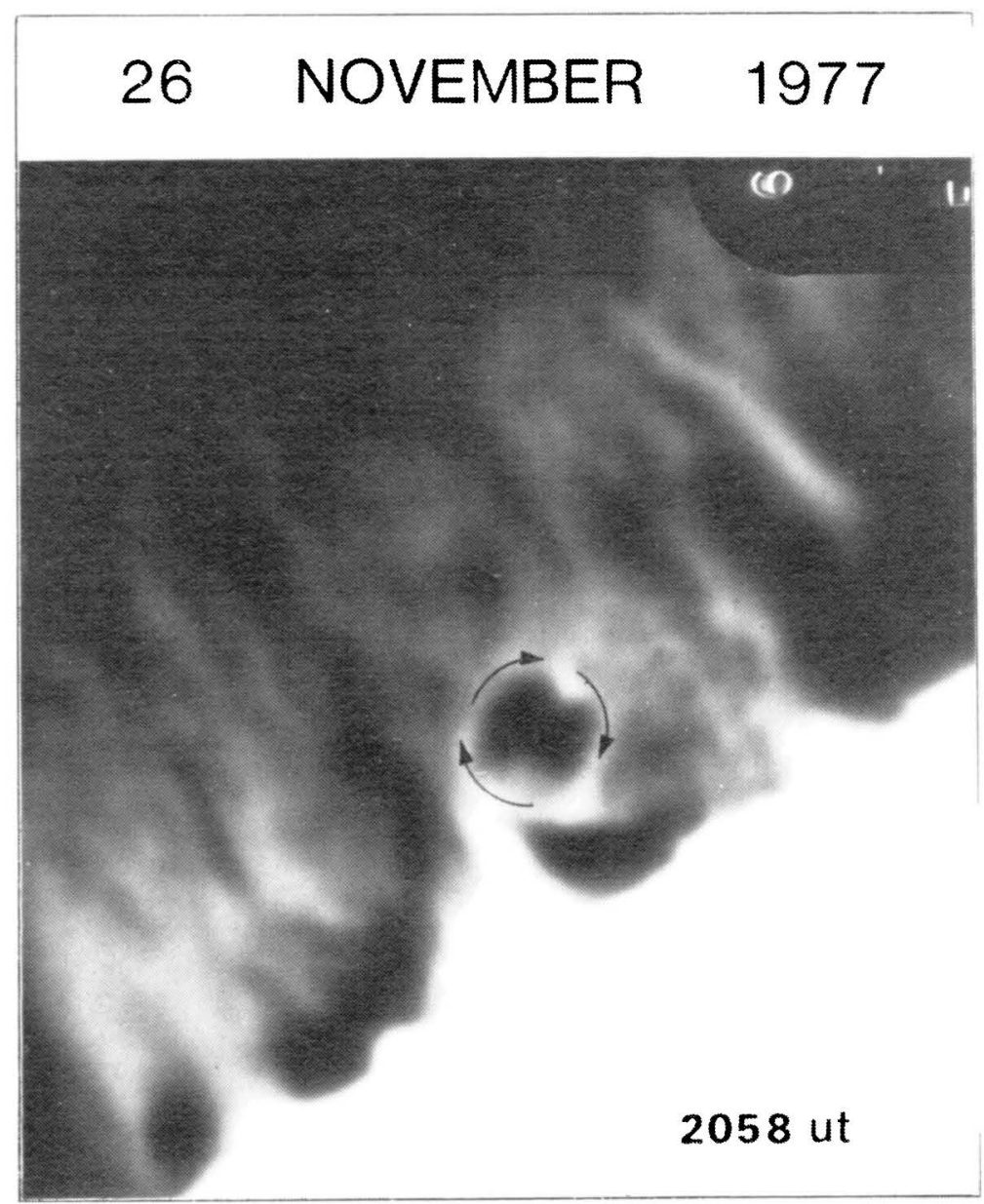

Fig. 2. A frame later in the day (20:58 UT) showing a complete eddy.

\section{August, 1978 (Figure 3.2)}

This prominence (NE) showed continuous rotation in proper motion about an axis perpendicular to the limb, as shown by arrows in Figure 3.2. If the material is truly rotating about such an axis, we should see blue shift at one end and red at the other. The many smaller eddies in the Doppler picture (Figure 3.2b) make this hard to see. The motion initially involved about half of the prominence, but more and more of the material participated in the rotation until the whole of the prominence was involved. The initial rotation period was about $40 \mathrm{~min}$. As most of the prominence became involved the period increased to about $150 \mathrm{~min}$. Upward motion of material was visible in the main trunk.

On the next day the prominence was still present but in a significantly different conformation, and composed of dense as well as diffuse parts. It disappeared sometime between the end of our coverage on the 5th and daybreak on the 6th of August. 


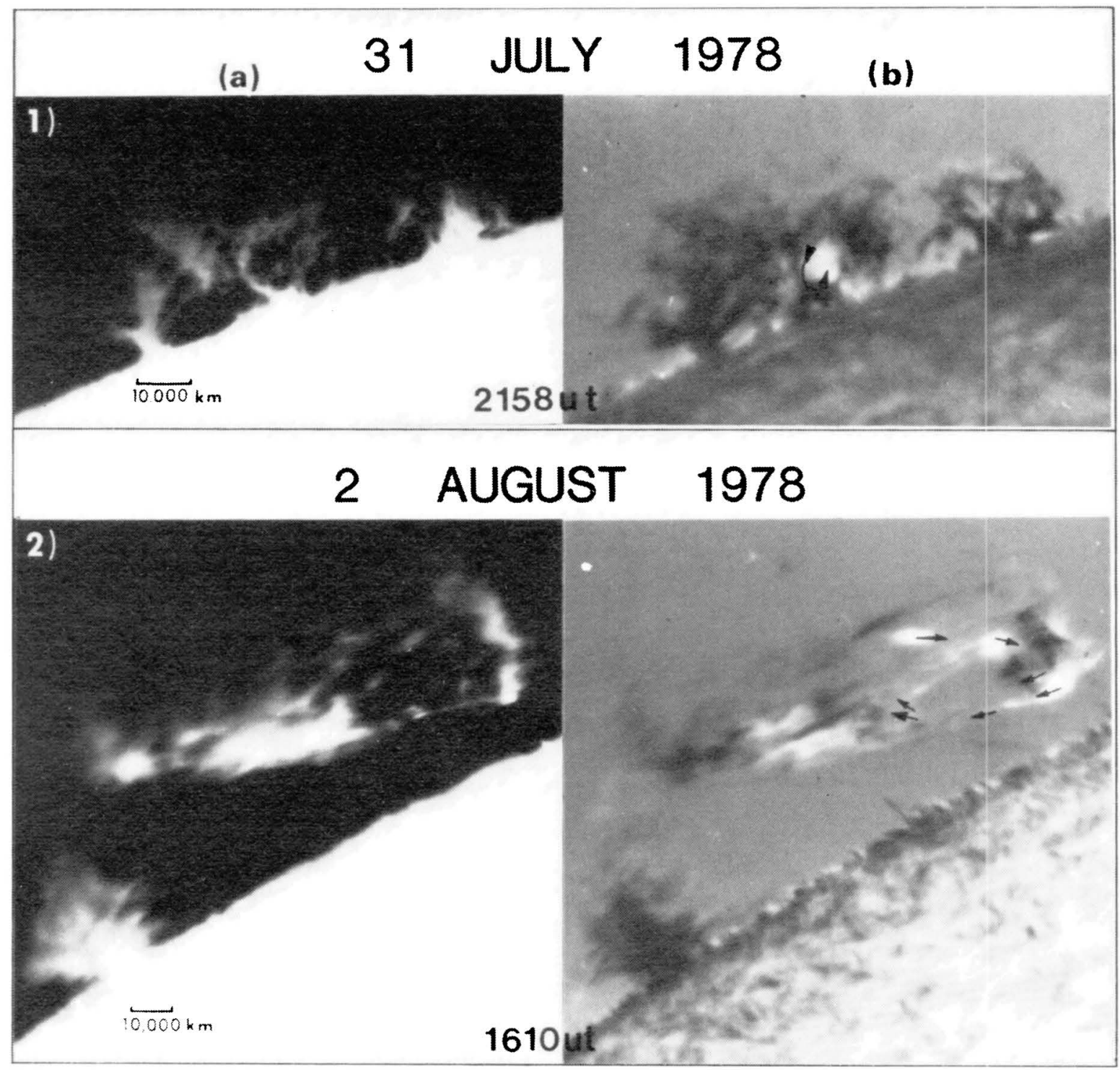

Fig. 3. (a) $\mathrm{H} \alpha$ line-center and (b) $0.25 \AA$ Doppler subtractions of the prominences of: (1) $31 \mathrm{July}, 1978$ at 21:58 UT, and (2) 2 August, 1978 at 16:10 UT. Black is blue-shifted and white is red-shifted.

\section{August, 1978 (Figures 4 and 5)}

For four days, 15-18 August, 1978, we observed part of this extended polar filament crossing the northeast limb (Figure 4). There was some up and down flow in a localized area $(\mathrm{N}$ end) on the 15 th but no radial motion was obvious. On the 16th and 17 th there was little motion. However, on the 18th the north end of the prominence rotated continuously.

Around 16:00 UT we saw proper motion rotation about an axis toward the observer and radial velocity rotation about an axis to the left, tangent to the surface (Figure 4.1). At 18:00, a new eddying motion propagated up through the prominence, involving the whole prominence by 19:00 UT (Figure 4.2). This broke up into complex eddies, and the Doppler frame shown in Figure 4.3 at $\sim 20: 00$ UT now shows rotation about a 


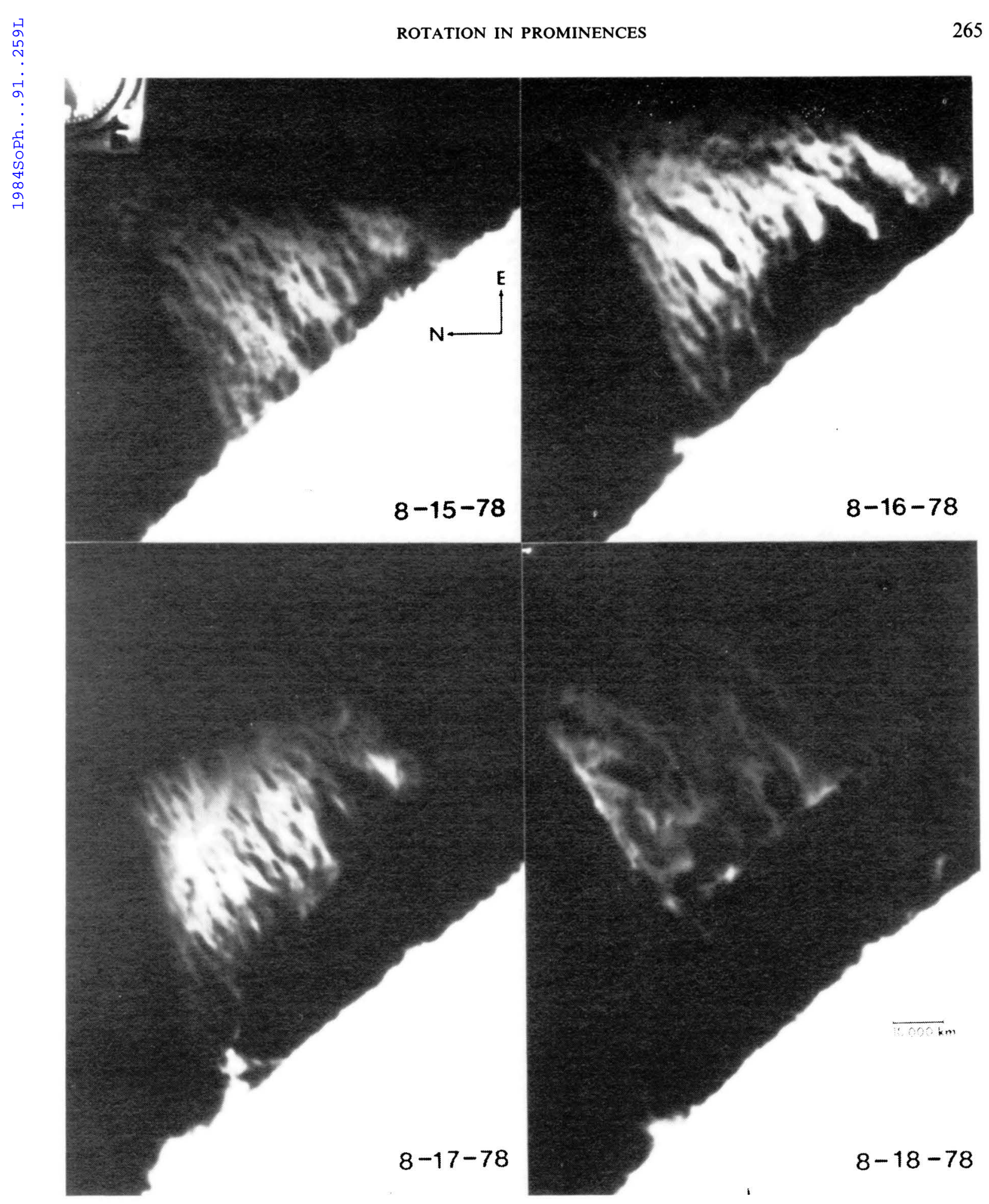

Fig. 4. $\mathrm{H} \alpha$ line-center from 15 to 18 August, 1978 showing the relatively stable conformation of the prominence which showed the dynamic rotation on 18 August, 1978 (Figure 4).

vertical axis. By 23:00 the prominence gave the appearance of breaking up, having risen $24000 \mathrm{~km}$ since 20:00 UT, but it did not rise much higher and was still on the limb on the following day. 


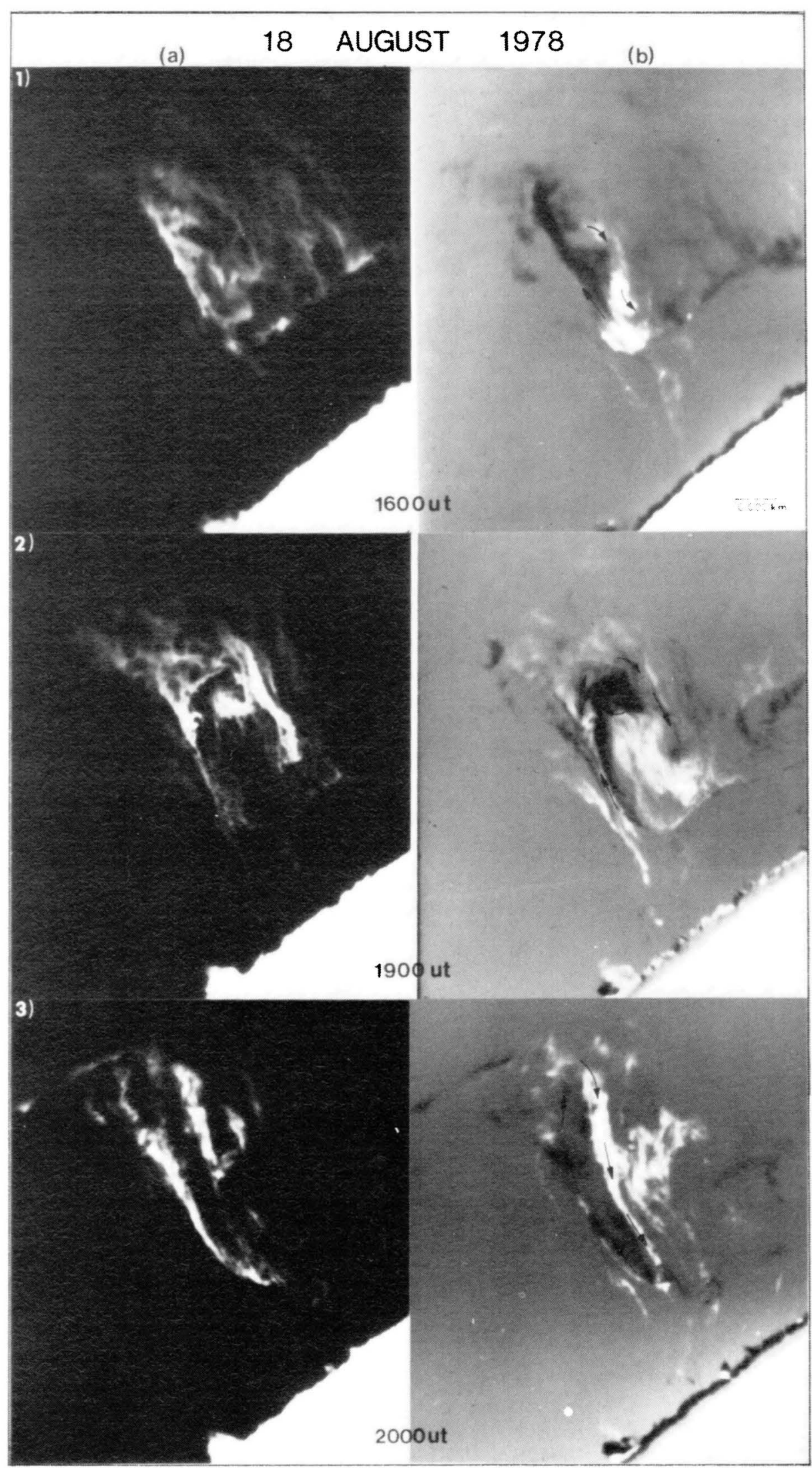

Fig. 5. Column (a) shows line-center and (b) shows $0.5 \AA$ Doppler subtractions of the 18 August, 1978 prominence at 16:00,19:00, and 20:00 UT. Again, in the dopplergrams, black shows blue-shifted material and white indicates red-shifting. 


\section{Discussion}

Although proper motions can be discounted as a phase or excitation phenomenon, Doppler shifts can only be produced by actual motion, so Öhman's idea that these objects are rotating was correct. The remarkable aspects of the rotation is that it goes on and on. This appears impossible in the light of our concept of lines of force. Since prominences must be held up by the lines of force threading them, any rotation must be shared by those lines. Eventually they should wind up and the rotation should cease, unless the lines of force reconnect at the axis of rotation. So the continual rotation may be viewed as evidence for reconnection on a time scale of hours. The kinetic energy of the rotating material (about $1 \mathrm{erg} \mathrm{cm}^{-3}$ for $N=10^{11}$ ) is greater than that of the $1 \mathrm{G}$ field thought to be present in such objects, so it easily can wind up the lines, but the rotation itself must be a magnetic phenomenon; one hardly would expect it to come from convection. As noted above, rotation is common in erupting prominences, but that can be ascribed to unwinding of twisted fields (i.e. we push the twisting back into the unknown past).

\section{Acknowledgements}

This work was supported by the NSF under ATM-8211002 and by NASA under NGL 05-002-034.

\section{References}

Leighton, R. B.: 1959, Astrophys. J. 130, 366.

Malville, J. M. and Toot, G. D.: 1982, Solar Phys. 80, 279.

Öhman, Y. (ed.): 1968, Nobel Symp. 9, 95.

Öhman, Y.: 1969, Solar Phys. 9, 427.

Rompolt, B.: 1975, Solar Phys. 41, 329. 